\title{
Influencing Factors in Prioritized Model of Patient Treatment with Mobile Computed Tomography Scan for Medium-Sized Hospitals in Thailand
}

\author{
Chamaiporn Sudasna Na Ayudhya ${ }^{1}$, Achara Chandrachai ${ }^{2}$, Kriskrai Sitthiseripratip ${ }^{3}$, Sombat \\ Muengtaweepongsa ${ }^{4}$, and Nattee Niparnan ${ }^{5 *}$ \\ ${ }^{1}$ Chulalongkorn University, Technopreneurship and Innovation Management Program \\ Bangkok, Thailand \\ ${ }^{2}$ Chulalongkorn University, Faculty of Commerce and Accountancy, Chulalongkorn Business School \\ Bangkok, Thailand \\ 3 National Metal and Materials Technology Canter, Head of Biomedical Engineering Research Unit, Thailand Science Park \\ Pathum Thani, Thailand \\ ${ }^{4}$ Faculty of Medicine, Thammasat University, Paholyothin Rd., Klonglaung, \\ Pathum Thani, Thailand \\ ${ }^{5}$ Chulalongkorn University, Faculty of Engineering, Department of Computer Engineering, Thailand, 10330 \\ ${ }^{*}$ Corresponding author's email: nattee.n [AT] chula.ac.th
}

\begin{abstract}
The purpose of this study was to determine the factors influencing the prioritization model for the treatment of patients with mobile computed tomography $(\mathrm{mCT})$ scanning. The main research process entailed value innovation product planning (VIPP), which focuses on three steps: idea generation; idea screening; concept testing and development. This was applied research comprising primary and secondary data, based on contextual research in conjunction with in-depth interviews from neuro-medical and senior professional level staff. Through the VIPP process, here were show three factors affecting the calculation in the scheduling model: the reservation queue to use $m C T$, the distance between the hospitals and the severity or waiting time of the patients. The results indicate that it is necessary to consider these factors in developing and testing scheduling equations using a specially developed computational program that can handle scheduling problems with limited resources to support the determination of mCT space and schedules, next phase.
\end{abstract}

Keywords-Mobile CT scan, new product development process, queuing, limited resource

\section{INTRODUCTION}

On World Stroke Day in 2016, the Health Systems Development Group, Bureau of Non-Communicable Diseases, and the Department of Disease Control noted that Paralysis or stroke is a global health problem. Currently, there are 17 million stroke patients worldwide; of these, 6.5 million people have died from stroke and 26 million people have survived stroke. Many stroke survivors are affected by physical, mental, social, and economic loss. In Thailand, stroke death rates per 100,000 populations in the country from 2013 to 2015 are 36.13, 38.66 and 42.62, respectively. From the above information, it can be seen that stroke mortality in Thailand has increased every year, as in the world overall, and the case of Thailand shows that stroke is a threat to the global population. In 2016, the World Stroke Organization (WSO) focused on the prevention and control of stroke in three main areas: awareness (Awareness), access to public health services (Access), and action to control stroke (Action) [1].

Of these three aspects of stroke prevention and control, the second, namely promoting access to health services, corresponds most closely to the main aim of this study. Studying the past literature on issues related to access to health services, it is apparent that there are gradually increasing numbers of studies and developments in this area. According to early research from 2009 to 2010 , it is necessary to reduce the risk of disability or death among stroke patients. In this regard, it is well known that treatment of acute paralysis with a recombinant tissue plasminogen activator (rt-PA) as soon as possible within three hours of the onset of symptoms is critical to reduce the risk of disability or death. The proportion of stroke patients with atrial fibrillation or stroke treated with rt-PA in the United States (US) is relatively small at 1.8 $3.0 \%$ due to patients not arriving at hospital swiftly enough. Walter et al. [2] addressed this problem, requiring screening and prompt treatment within a given treatment time. Starting in 2009, their research entailed modifying an ambulance to 
develop a specialized mobile stroke unit (MSU, or "Stroke Ambulance"), including the capability to undertaken a computed tomography (CT), a diagnostic system for stroke, and a long-distance therapy system (telemedicine) that has the potential to contact a physician or specialist to diagnose a patient's symptoms based on CT images showing patients with ischemic stroke or hemorrhage stroke enabling initial treatment in the MSU by a doctor or specialist.

$\mathrm{Na}$ Ayudhya et al. [3] found that of the leading neurosurgeons in Thailand, $85 \%$ were interested in the provision of $\mathrm{mCT}$ services in the operating room (OR), intensive care unit (ICU), and emergency room (ER). As a result, the needs of neurosurgical physicians were studied to determine factors affecting the model of provision and prioritize the treatment of mCT patients in medium-sized hospitals in Thailand. Among the issues affecting the ability to develop a scheduling equation that is specific to the requirements and the particular conditions concerning the provision of mCT services in approximately 83 medium-sized hospitals in Thailand are the delimited area and limited resources [4].

\section{BACKGROUND AND RESEARCH QUESTION}

A research study "Influencing Factors in Prioritized Model of Patient Treatment with Mobile Computed Tomography Scan for Medium-Sized Hospitals in Thailand" was developed to determine the factors that affect the scheduling model through the new product development (NPD) process, and to design and develop a model to deal with scheduling problems related to mobile computed tomography (mCT) scanning in the case of limited resources. The model created is unique in that the research brought the problems and needs of the physicians into the process of creating the optimal model. To establish the appropriate research methodology, the literature review covers two areas: theory of the NPD process and the current status, problems, acceptance, and performance of mCT.

\subsection{Theory of New Product Development process (NPD)}

Product planning is the early stage in the process of NPD, and forms the basis of guidelines or principles for innovators or NPD teams in developing products to meet or satisfy the needs of consumers. When developing new products, whether tangible or intangible, such as services, they will be realized and ultimately will be sustainably accepted by consumers or not; this depends on the product planning process. The first phase of this process is chaotic and confusing, so it is often referred to as the "fuzzy front end" (FFE) of innovation. This study reviewed five models of product development and selected one of the five major processes.

Based on the review of the five product planning models shown in Table 1, it is evident that the five core NPD processes differ slightly, but broadly the five front-end models consist of five steps: opportunity identification, idea generation, idea screening, concept testing and development, and business analysis. The results of front-end processing are the project mission statement and product plans that are ready to be delivered to the next phase of product development, sometimes called the back-end process, which also comprises three main steps: product testing and development, test marketing, and commercialization. The five product planning processes are slightly different; in this case, the study employed the fifth model, value innovation product planning (VIPP), because this research is related to innovative products and services. In the product planning phase, it is necessary to determine the target audience and the suitable study method, and then choose the right information at the right time for each step to ensure that the project mission statement meets the target needs and is accepted by the real market in the future.

Table 1: Five different types of product planning [5-9].

\begin{tabular}{|l|l|l|l|}
\hline Year & \multicolumn{1}{|c|}{ Authors } & \multicolumn{1}{|c|}{ Model } & \multicolumn{1}{|c|}{ The main issue } \\
\hline 1982 & $\begin{array}{l}\text { Booz, Allen \& } \\
\text { Hamilton }\end{array}$ & BAH Model & $\begin{array}{l}\text { The model focuses on strategic planning for new products, and is suitable for } \\
\text { non-profit executives in small and medium-sized businesses as it is a simple, } \\
\text { uncomplicated process. This model must be targeted, employing tactical } \\
\text { strategy in NPD until the product idea is ready for process development, } \\
\text { market testing, bringing to the commercial market, and monitoring of feedback } \\
\text { from consumers. }\end{array}$ \\
\hline 1997 & $\begin{array}{l}\text { Khurana, A. } \\
\text { Rosenthal, S. R. }\end{array}$ & $\begin{array}{l}\text { Model of Front } \\
\text { Ends }\end{array}$ & $\begin{array}{l}\text { The model focuses on making the product planning process as successful as } \\
\text { possible for large organizations by requiring a link between three factors: the } \\
\text { business strategy, product strategy, and product-specific decisions. }\end{array}$ \\
\hline 2002 & Koen et al. & $\begin{array}{l}\text { New Concept } \\
\text { Development } \\
\text { Model } \\
\text { (NCD Model) }\end{array}$ & $\begin{array}{l}\text { The main goal of the NCD model is to provide methods, tools, and techniques } \\
\text { that are appropriate and effective for managing the product planning process. } \\
\text { This model consists of three major parts that influence the innovation process } \\
\text { from the very beginning to the final commercialization phase, the engine, the } \\
\text { controllable activity elements and organizational capabilities and the outside } \\
\text { world. }\end{array}$ \\
\hline Cooper, R. G. & $\begin{array}{l}\text { In the model, the product innovation process from idea to launch is similar to } \\
\text { BAH model (Booz et al. 1982), but in this style, there is a gate in screening } \\
\text { each process that is passed or failed (go-kill), and ideas that are not viable are } \\
\text { returned to the previous process for re-synthesis and re-evaluation. }\end{array}$ \\
\hline
\end{tabular}




\begin{tabular}{|l|l|l|l|}
\hline Year & Authors & \multicolumn{1}{|c|}{ Model } & \multicolumn{1}{c|}{ The main issue } \\
\hline 2010 & Israsena P. & $\begin{array}{l}\text { Value Innovation } \\
\text { Product Planning } \\
\text { Model } \\
\text { (VIPP Model) }\end{array}$ & $\begin{array}{l}\text { The goal of the VIPP process is to encourage the organization to create value } \\
\text { innovations and solve product development issues that are not consistent with } \\
\text { the value proposition of the target audience, thus leaving no market for new } \\
\text { products. This model is based on the choice of study methods and uses the } \\
\text { necessary information concerning the target audience in a timely manner for } \\
\text { each process step. }\end{array}$ \\
\hline
\end{tabular}

VIPP classifies projects based on four categories: new look and feel, new solution, new usage, and new value. These differ in terms of how they work and how they are linked within different sub-processes. As this study concerns product development that responds to new applications that have not yet been implemented, or develops a product to facilitate and meet the unmet needs of users, the choice was made to use the VIPP model, addressing new usage through the following product planning phases:

Starting from opportunity identification, at this stage, it is important to gain an understanding of the problems and limitations in the use of original products through interviews and observations in the real context, focusing on the lead user(s). Second, idea generation, this stage entails conducting an exploration of all aspects related to the objectives and scope of the project, focused on the relationship between value and use of the product. Third, idea screening, this stage the number of options is reduced, while simultaneously improving the quality of ideas. Fourth, concept testing and development, this is the new usage project focuses on conceptual testing, undertaking a new value perception test to determine priorities from the perspective of the target group. However, as the target audience will not be the same as those with needs different from the original product, it must be imaginative and may not be accurate. Therefore, the semantic differential can be used to evaluate the attitudes of target groups, possibly with media such as the service blueprint, abstract prospective scenario, video, etc. Finally, business analysis, at this phase feasibility studies are conducted, for new usage projects, however, the results are highly uncertain due to the analysis being done in a predictable manner based on a new market that has not actually arisen.

\subsection{Current Status, Problems, Acceptances and Performance of mCT}

In 2010, Jin Wook Kim et al. [10] undertook research on mCT scanning in Korea. Visualization during surgery is very important, but this was a limitation of the neuronavigation system available in 2010 , which could not display the results of "brain shifts" resulting from a loss of cerebrospinal fluid (CSF) or cranial wounds during surgery. Therefore, surgical imaging is essential for the accuracy of the neuronavigation system. In their study, Jin Wook Kim et al. (2010) compared the use of intraoperative magnetic resonance imaging (iMRI) with $\mathrm{mCT}$ and found that using CereTom, an mCT scanner manufactured by Neurologica in the US, there is no need for any special measures or tools to address issue that prevent taking X-rays in the operating room as the patients lie in the usual position on a headrest attached to the bed frame. The simple, easy-to-use design of the mCT scanner means it can easily be moved to an OR, ICU or ER room. Based on Jin Wook Kim et al.'s [10] study in Korea concerning the effect of mCT on clinical use in the early stages of adoption, Jin Sue Jeon et al. [11] collected experience data on the use of $\mathrm{mCT}$ during operations and bedside scans over a period of three years from January 2009 to December 2011, using four mCTs in operation in Korea since late 2008. As a result of the introduction of $\mathrm{mCT}$ as a tool in the $\mathrm{OR}$, neurosurgeons were found to be able to manage decision making in the ICU in a more timely and secure manner.

Subsequently, Dietrich et al. [12] undertook further research based on the work of Walter et al. (2010). They studied the subjects of prehospital treatment, examining expenditure and undertaking an economic assessment. A stroke care strategy was developed specifically for emergency use by the MSU. The use of the MSU was found to be associated with a reduction in the number of patients experiencing delayed thromboembolism; however, it was also criticized for incurring unacceptable costs. Thus, this study investigated the correlation between the direct costs of care for stroke patients and the introduction of prehospital treatment. Over a period of one year, the study found that prehospital treatment can save direct costs under five scenarios based on three parameters: the influence of emergency medical personnel, the influence of distance on operation, and the influence of population density. Beyond that, the research also showed when the number of staff or emergency medical staff is reduced along with a higher population density the benefit ratio increases.

In the context of Thailand, Phayathai Hospital [13] officially established an MSU with an innovative ambulance stroke patient treatment that is the main device for its operation. In 2015, it was the first such ambulance in Asia and the fifth in the world. Dr. Surat Boonyakarnkul and his team studied the success of use of MSUs both in Germany and the US, finding that it is possible to treat stroke patients faster than in conventional ambulances using specialized equipment and modern communication technology. In an acute stroke unit ambulance, there will be X-ray computer brain scanning capability and CT scanning for stroke, enabling immediate diagnosis. Moreover, an on-board mobile laboratory can detect the need for blood transfusions in treating cerebrovascular disease before reaching the hospital, as well as having medications and supplies needed to treat stroke, especially intravenous thrombolytic drugs. Finally, it also has tools to communicate over the Internet and through a video conferencing system to provide online treatment. With this 
combination of equipment, technology, and the expertise of specialized medical professionals, Phayathai Hospital has been able to increase the efficiency of treatment for stroke patients. In the case of obstruction or bleeding in the brain, the patient will be diagnosed immediately upon arrival by brain imaging using a CT scanner installed in the unit and patients are also assessed via the internet to determine whether thrombolytics are available or to prepare a physician and operating room when the patient arrives at the hospital; then, the physician treats the patient with the fastest method of blood clotting or in the case of urgency through surgery.

In 2016, Agrawal et al. [14] conducted a study on mCT use in patients with bedside scans. They undertook more than 67 months of data collection from June 2009 to January 2015, obtaining up to 10,000 cases in the surgical department at hospital level 1 in a trauma center in India. Scans are typically needed in patients with symptoms of traumatic brain injury (TBI) to evaluate and manage such head injuries. Particularly in the case of multiple injuries, the blood flow is not stable and there is a line in the blood vessels, making it difficult to move the patient to a CT scan. In this study, for the first 1,000 patients, the researchers used the number of times the CT scan was performed, the time taken for imaging, and the stoppage time. Same year, John et al. [15] studied mCT scans in terms of current status and future prospects. Hence, the advancements in biomedical engineering have led to developments in sharpness and image quality with reduced radiation, resulting in portable or mobile CT units. This is another important advancement and is especially useful in assessing the severity of patient conditions in the ICU through bedside scanning. As mentioned above, there has been a paradigm shift in inserting $\mathrm{mCT}$ in ambulances to manage stroke, which is especially valuable because time is critical for ischemic stroke or clogged arteries. John et al.'s [15] study investigated two cases in Saarland and Berlin, examining the safety and feasibility of prehospital treatment, and demonstrating that MSUs can increase the rate of recognition of thromboembolism in patients and reduce the duration of treatment compared to regular treatment. MSUs can also help develop a patient-specific screening system to send patients to specialized hospitals.

The latest research related to the benefits and challenges of MSU, namely that of Fassbender et al. [16], argues that in the management of cerebral stroke, "time is the brain." Based on past studies, attending to patients quickly rather than waiting for them to be hospitalized, improves the outcomes of cerebral palsy. This strategy is based on using an MSU ambulance with a photographic tool, specific laboratories, and Internet access for remote treatment (telemedicine) with appropriate medicine. The results of an outpatient stenting study were consistent with the reduction in delays in hospital admission prior to thromboembolism and patient screening, enabling access to appropriate hospital admission, whether to normal units or central stroke treatment centers.

Despite all the above research, analysis of the current status, problems, acceptance, and performance of mCT scans, little research has been published and it can be observed that existing works pertain to developed countries such as the US, Germany, and Korea, which have studied and developed MSU CT scanning together with other support systems. As mentioned, from 2008 until now, looking at Asia, results have been published from India, studying the benefits of mCT as a bedside diagnostic tool for patients, and from Thailand, showing a good response in terms of the successful application of MSUs. This study is of the view that research into the use of mCT is of value in diagnosing stroke as well as aiding decision makers in treating stroke patients under limited scheduling conditions to support the definition of areas and usage scheduling, which is another issue that is important for the future development of MSUs.

\subsection{Research question}

This research addressed one question: What are the factors that affect $\mathrm{mCT}$ service ranking for patients in different hospitals in different areas? It considered resource conditions related to limited $\mathrm{mCT}$ machine usage and studied three topics: idea generation, idea screening, and concept testing and development.

\section{METHODOLOGY}

This research addressed one question: What are the factors that affect mCT service ranking for patients in different hospitals in different areas? It considered resource conditions related to limited $\mathrm{mCT}$ machine usage and studied three topics: idea generation, idea screening, and concept testing and development. The study comprised applied research based on a contextual examination in conjunction with in-depth interviews based on the product development planning phase in VIPP aimed at achieving a project mission statement and product plan.

\subsection{Population and Sample}

The population of the study was divided into two groups according to the sub-procedure of the VIPP model. The first group concerned the idea generation phase, incorporating one-year statistical data of stroke patients retrospectively collected in 2015, from January to December, based on gender, age, NIHSS score, and symptom classification of stroke patients from hospital A. This yielded 765 data sets for analysis. The data thus collected were then considered together with the contextual data (contextual research), summarizing factors for presentation to the second population group. The second group related to idea screening and included neuro-medical and senior level professionals involved in working with stroke patients at hospital A; these were the lead users of the data collected from the first population. This group was asked to provide additional information through in-depth interviews based on the factors drawn from the statistical analysis of data from the first population group. 


\subsection{The instrument used in research}

Three kinds of instrument were used for data collection in the idea generation step. The first was an observation sheet collecting data on the time of travel of the $\mathrm{mCT}$ from one point to another. Real data were collected through observation under real conditions in the context of use to provide the whole picture of the actual situation. The second was a data collection sheet for primary data collection from the stroke unit operating files in the hospital as a case study; information was collected on gender, age, the National Institutes of Health Stroke Scale (NIHSS) scores, and the statistical classification of patients [17]. The last was an observation sheet with additional topics for in-depth interviews in primary data collection based on the observation conducted while the researcher was embedded with the lead user for about a month in a case-control study and also attended "Basic Stroke" sessions organized by Thammasat University Hospital for five days.

\subsection{The process used in research}

This study used VIPP as the main research methodology. This comprised the usual five steps, but this paper focuses on three of these: idea generation, idea screening, and concept testing and development as details below.

Opportunity identification: The study examined demand for, and expectations and acceptance of new services using mCT through in-depth interviews with the 21 leading neurosurgeons of the country, based on the results of research by Sudasna Na Ayudhya et al. [3].

Idea generation: The three components were studied together: the first was based on the total time of travel of the mCT from one point to another as detailed above; the second concerned primary data on statistical information collected at the hospital as a case study in conjunction with the interviews; the third component comprised embedding in the lead user through "Basic Stroke" training held on January 16-20, 2017, at Thammasat University Hospital.

Idea screening: The information from the three components in idea generation step were taken into consideration and summarized into constant value or random variable that were expected to affect scheduling equations. Then, these were presented to lead users (population group 2) and in-depth interviews were undertaken to receive feedback and develop a guideline for the selection of variables to be grouped into the main factors used in the scheduling equations. The scheduling equations aimed to calculate the order of mCT services for patients at different hospitals and in different areas that would be most effective under conditions with limited resources.

Concept testing and development: In terms of the main factors used to calculate the scheduling equations, in the idea screening step the research took the key factors into consideration in relation to the decision to diagnose symptoms or to provide patient treatment. Lead users were asked to identify which factors should be considered main factors or secondary factors, or whether all factors should be viewed as equally important through in-depth interviews. The entire development of the scheduling equation was intended primarily to meet the needs of physicians who want to move the machine to the patient and at the same time shorten the waiting time for the patients.

\section{RESULTS AND DISCUSSION}

This research studied an NPD process, focused on three main aspects - idea generation, idea screening, and concept testing and development - to obtain factors influencing the prioritization model for the treatment of patients with mCT scanning in medium-sized hospitals in Thailand as the following details.

\subsection{Idea generation}

The researcher has studied the three components together in this step as follows. The first component examined was the time of travel from one point to another using data collected at a special dental clinic, the Faculty of Dentistry, Chiang Mai University, over a period of three days. The mCT was moved from the National Metal and Materials Technology Center (MTEC) in Pathumthani province and transported to the special dental clinic in Muang District, Chiang Mai province. The mCT scanner was then unpacked, taken to the room in which it was to be used, and set up. It was then ready for photographic scanning of patients and displaying the scanned images on the case display, case by case. Furthermore, the researcher participated in a workshop on the use of mCT scanners with dentists and nurses from this Faculty. The observation sheet and in-depth interviews with specialists yielded 13 conditions related to time for calculation in the scheduling equations, as shown in Table 2.

Table 2: Pre-interview data from idea generation step

\begin{tabular}{|c|c|c|}
\hline$\overbrace{\text { Information Status }}^{\text {Conditions }}$ & mCT's conditions & Hospital's conditions \\
\hline From observation & $\begin{array}{l}\text { 1. Set time before moving } \\
\text { 2. Time spent to transport vehicles } \\
\text { 3. Time spent moving from car } \\
\text { 4. Time spent moving into hospital } \\
\text { 5. Time spent moving to the operating }\end{array}$ & $\begin{array}{l}\text { 1. Number of hospitals } \\
\text { 2. Distance of each hospital to go } \\
\text { 3. Disease to be diagnosed } \\
\text { 4. Number of patients seeking active } \\
\text { 5. Severity of the disease }\end{array}$ \\
\hline
\end{tabular}




\begin{tabular}{|l|l|l|}
\hline & $\begin{array}{l}\text { room or other position in the } \\
\text { hospital }\end{array}$ & 6. Patient waiting time \\
6. Set time at the room or area to use & \\
7. Duration of use & \\
\hline
\end{tabular}

The second component comprised the primary data collected from the stroke unit operating files in the hospital as a case study, including information on gender, age, the National Institutes of Health Stroke Scale (NIHSS) scores, and the statistical classification of patients. In all, 765 data sets were collected retrospectively in 2015 to determine frequency and the relationships of all data collected for the group of patients, which were then presented to experts in the next step. As can be seen from Figure 1, shows that for each of the NIHSS score ranges, the highest numbers of patients were aged over 65 years $(5 \%, 14 \%, 16 \%$, 6\%, and 5\%, respectively), followed by the 56-65 and 46-55 age groups, respectively. Among patients under the age of 45 years, the average rate was $9 \%$, but the scores for patients aged 46-65 years showed considerable variability, ranging from no stroke (0) to severe stroke $(21-42)$, at $3 \%, 6 \%, 6 \%, 2 \%$, and $0.4 \%$ of all patients, respectively, with a prevalence of patients aged 46-65 years at NIHSS scores $0-4$ and 5-15.

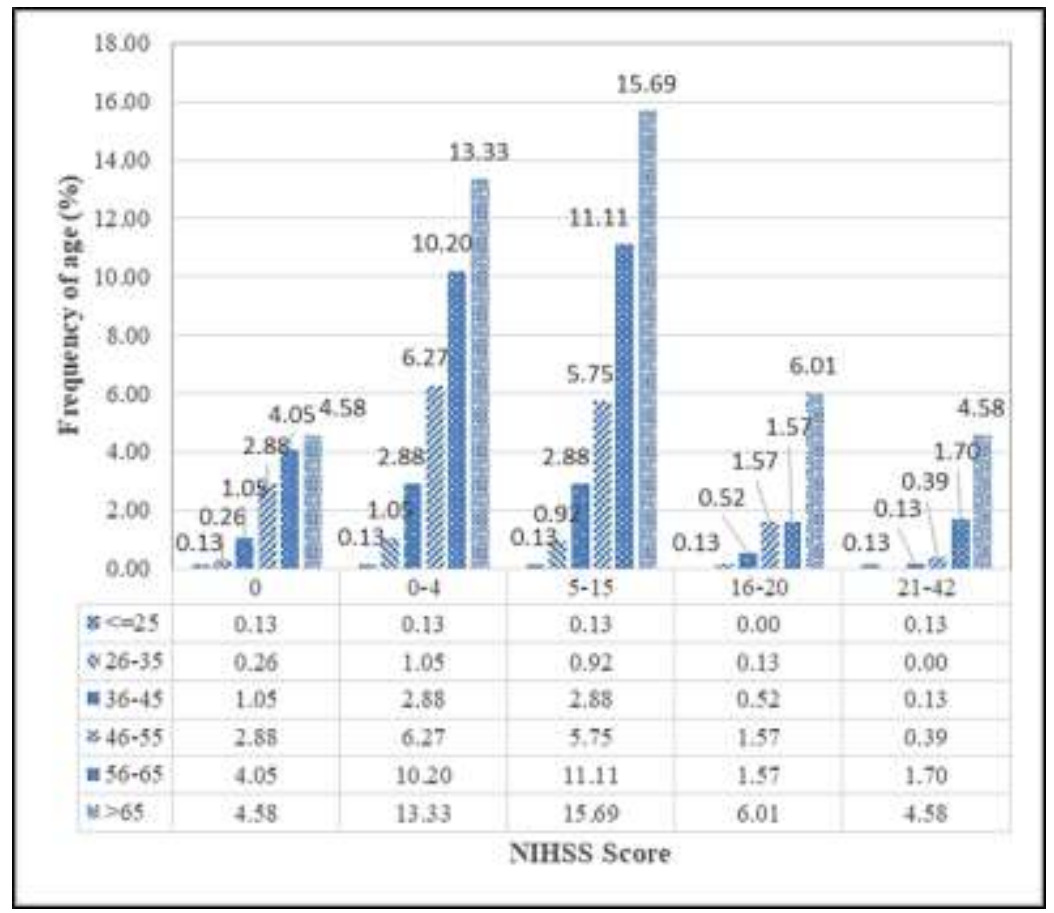

Figure 1: Frequency of stroke patients classified by age and symptoms using NIHSS scores from the stroke unit operating files in hospital A. One-year statistical data retrospectively collected in 2015.

In terms of patient classification, this was based on criteria for screening patients, i.e., whether on the stroke fast track in the hospital case study or not. The hospital determines the time from the onset of symptoms to the patient's arrival at the hospital. If the duration is less than 4 hours 30 minutes, the patient will be screened for immediate stroke fast tracking in the hospital. The results of Table 3 show that by 2015 , almost $58 \%$ of patients were eligible for fast tracking but $42 \%$ were not.

Table 3: Patient classification data based on screening criteria for patients, whether fast tracked for stroke or not at hospital A).

\begin{tabular}{|c|c|c|}
\hline Results & Frequency & Percentage \\
\hline Meets criteria & & 58.17 \\
\hline Does not meet criteria & 445 & 41.83 \\
\hline
\end{tabular}

The third component was contextual observation, based on embedding with the lead user for about a month in a case-control study and also attending the "Basic Stroke" training organized by Thammasat University Hospital for five days. The observation followed the hospital's approach to stroke patients from the patie nt's arrival at the hospital until they were diagnosed and treated according to the severity of the symptoms. In addition, the "Basic Stroke" training covered various aspects of knowledge, encompassing the definition of the disease, the physical characteristics of the brain in the normal state, observable symptoms of stroke, methods of assessment and 
meanings of all NIHSS scores, symptom correlations detected by NIHSS scores, and the physical characteristics of the brain in abnormal conditions. Moreover, the researcher also observed the way in which physicians and nurses use the association of symptoms to pinpoint a deficiency in the brain before a patient is screened for a CT scan; this initial indication helps prepare the patient for treatment faster and more efficiently.

\subsection{Idea screening}

The three components of the idea generation process - the time of travel from one point to another, the primary data collected from the stroke unit operating files, and the observational and in-depth interview data from the embedding with the lead user-were taken into consideration to define the constant variable and those expected to affect the calculation in the scheduling equations as shown in Table 4. Based on the observational, pre-interview data shown in Table 3, six hospital conditions and seven mCT conditions were calculated. No matter how complex the journey was, these sets of constants remained the same, representing the time to install and move the mCT from the MSU to the destination hospital. The random variable have already been discussed, as presented to neuro-medical and senior professional level staff at the hospital to obtain suggestions and guidelines for the selection of which random variable are most important and which ones are ranked as secondary through in-depth interviews. In addition, they confirmed that the seven constant values did not affect decision making in ordering services for stroke patients as shown in Table 4.

Table 4: Factors influencing the results of the prioritization model from the idea screening step.

\begin{tabular}{|c|c|c|}
\hline Information Status & Constant values (Standard time) & Random Variable \\
\hline $\begin{array}{l}\text { After the interview } \\
\text { (From indepth- } \\
\text { interview) }\end{array}$ & $\begin{array}{l}\text { 1. Set time before moving } \\
\text { 2. Time spent to transport vehicles } \\
\text { 3. Time spent moving from car } \\
\text { 4. Time spent moving into hospital } \\
\text { 5. Time spent moving to the operating room or } \\
\text { other position in the hospital } \\
\text { 6. Set time at the room or area to use } \\
\text { 7. Duration of use }\end{array}$ & $\begin{array}{l}\text { 1. Queue Reservation } \\
\text { 2. The distance between one hospitals to another } \\
\text { hospital. } \\
\text { 3. Severity of patient (Referring to the waiting time } \\
\text { of the patient) }\end{array}$ \\
\hline
\end{tabular}

\subsection{Concept testing and development}

At this stage, all 10 factors from the idea screening, shown in Table 4, were considered in terms of the decision of the lead user to diagnose the symptoms or to treat the patient. This aimed to identify which factors should be considered main and which secondary, or whether all the factors should be treated as equal in the scheduling equations developed with the main purpose "to reduce the waiting time for CT scan as much as possible." The concepts of testing and development were divided into two parts: seven constant values and three random variables, as detailed below.

Constant values were found to constitute the standard time of the calculation: Regardless of whether the beginning, intermediate, or last points change, this will not affect the standard time. These are the first four factors, number 1-3 and 6 , which will not change, except in terms of upgrading the external design of the machine to prepare it before traveling, or upgrading to enable it to be used faster or to enable a shorter warm-up time for the detector. There are two other factors, number 4 and 5, that the observational data obtained in the case study hospital were used to estimate the average of this standard time. The last factor in terms of constant values is duration of use, calculated based on the average time using data for patient alignment, the cycle of each scan at normal resolution, and the time between scanning and rendering for both bone and soft tissue. This last factor will change, yielding a shorter time when the software upgrades the rendering speed, or a longer time at a higher resolution.

In addition, three random variables were obtained from the idea screening process, number $1-3$, referring to the waiting time of the patient. These three aspects all change when the requirements for the $\mathrm{mCT}$ scan are being filled in, which means that the random variables must be derived solely from user input to the program developed. In the study, nurses as users supervised the appointments of patients for mCT scanning at each hospital. Thus, the queuing of reservations was the initial factor in terms of receipt of information from the system and was necessary to convert the queuing reservation data into a time that should be used. To do so, it is also necessary to have information on the severity of patient symptoms, defined in terms of three levels: Level 1, very severe, must be diagnosed or treated within 24 hours; Level 2, moderate to severe, needs to be diagnosed or treated within 72 hours; Level 3, less severe, must be diagnosed or treated within 168 hours [18]. To make this determination, reference was made to the frequency of patients at each level of the NIHSS scores (see Figure 1), as well as the results of the in-depth interviews in the idea screening process. The time for diagnosis or treatment was itself a number that would convert the data on queuing reservations to the time that should be used. 
Another important factor concerning random variables was the distance between one hospital and another; this is important in the scheduling equation. If users in different hospitals make requests to use the mCT scanning service simultaneously, the variables to be considered in addition to the booking sequence and the severity of the symptoms are the distance between the start of the service on that day and the location of the two hospitals that submitted simultaneous requests to use the service. The issue here is how the route should be formulated to answer the main objective of the scheduling equation "to reduce the waiting time for CT scans as much as possible." At this point, it is also necessary to convert units from distance to time for the scheduling equation to be developed; all the units in the calculation were the same, namely hour.

To ensure completeness and appropriateness for most applications, in developing the scheduling equation it is necessary to weight each of the variables for the three main parameters representing random variables. This was done in the form of linear equations based on self-ratings from a study of mortality and survival rates related to the implementation of CT scanning [19]. However, the source of scoring is not discussed here as the criteria are still the subject of a validation process. The scheduling equation of this study is deemed likely to be appropriate, consistent with the data and feedback obtained from the neuro-medical and senior level professionals in the idea screening process.

\section{CONCLUSIONS AND LIMITATIONS}

\subsection{Conclusions}

This paper has aimed to determine the factors influencing a scheduling model for the classification of patient care using mCT scanning for medium-sized hospitals in Thailand. It has addressed three processes: idea generation, idea screening, and concept testing and development. NPD process was the main research methodology employed in the front end, collecting primary and secondary data using observation sheets as a research instrument, in accordance with experience of the context, in conjunction with in-depth interviews based on the VIPP process. In terms of the research question, concerning factors that affect the mCT service ranking for patients in different hospitals in different areas, under resource conditions limited access to the mCT scanner was identified. Thence, three factors were found to affect the calculations in the scheduling equation: the reservation queue to use the machine, the distance traveled between hospitals each day, and the severity of symptoms or waiting time of the patients.

All the steps taken as a result of the above are significant starting points in terms of the choice of study methods. In particular, the study has used necessary information from the target audience in a timely manner for each step in the process step and avoided issues not consistent with the value proposition of this target audience, resulting in no market for new products. In the idea generation and idea screening phases, the choice was made to use in-depth interviews and observation in combination with secondary data collection. The results show that age and gender correlate with NIHSS scores. Based on these data, the researcher is of the view that further research should be conducted to determine the relationship between patients' age, gender, and NIHSS scores in case-control hospitals located in Central Thailand. In particular, it is necessary to determine whether age and gender should have a bearing on the decision to order diagnosis or treatment as this research does not take into account age and gender as main factors in the scheduling equation.

Another key observation is the fact that almost $45 \%$ of patients did not meet the criteria for stroke fast tracking, indicating patients or the general public may be little awareness of the initial symptoms of stroke, or may know but do not pay attention to their family or peers as they should. Thus, awareness needs to be raised so that in future, Thailand will be able to increase the percentage of those who meet the criteria, potentially reducing the number of patients who die from stroke and increasing the percentage of rt-PA treatment in hospitals in Thailand.

Based on the above observations, this study aimed to determine the factors that influence the ordering of services or the treatment of patients to develop a scheduling equation that was specific to the case study. Also, in future the pattern of the development of this scheduling equation can be adapted to take advantage of the management of activities focused on prioritizing services under conditions of constrained resources according to Thailand 4.0, which focuses on driving the economy through innovation by adopting creativity as a driving force and bringing about innovations in products and services.

\subsection{Limitations}

When considering mCT, one thinks first of scanning the brain. This research focuses on the provision of support for access to medical services with $\mathrm{mCT}$ for stroke patients in remote areas or medium-sized hospitals with inadequate budgets to procure equipment. According to the statistical data on patients with brain disease at hospital A, and considering the severity of the patient related to the waiting time to use a normal CT scan for stroke, it is very important to get an image within 25 minutes of arrival at the hospital, as recommended by the Prasat Neurological Institute [18]. The mCT medical research service may not be able to fully diagnose stroke patients in all cases, and thus it is necessary to adjust the conditions related to the patient's severity level from that used for stroke alone to a broader scope in determining the waiting period for a CT scan. After adjusting these conditions, the scheduling program developed can be used to calculate and rank the order of service in a way that does not conflict with the conditions of the medical service. 


\section{ACKNOWLEDGEMENT}

We gratefully acknowledge the 90th Anniversary of Chulalongkorn University fund (Ratchadaphiseksomphot Endowment Fund) for funding this research and we would also like to extend our sincere thanks to Dr. Nattaphol Uransilp, Neuro-medical, and Urai Kommarg, Senior Professional Level from Thammasat University Hospital for their assistance and permission to attend "Basic stroke" training that held on 16-20 January 2017, including an explanation on a description of medical terminology throughout the 5-day training.

\section{REFERENCES}

[1] Bureau of Communicable Diseases, "World stroke campaign 2016 about world stroke day 2016", Available at: http://www.thaincd.com/document/hot\%20news/WorldStrokeday2016.pdf, accessed 19 July 2017.

[2] Walter S, Kostpopoulos P, Haass A, Helwig S, Keller I, Licina T, et al.., "Bringing the hospital to the patient: first treatment of stroke patients at the emergency site", PLoS One, 5(10):e13758, 2010. DOI: 10.1371/journal.pone.0013758.

[3] Na Ayudhya CS, Chandrachai A, Sitthiseripratip K, Niparnan N, "Demand of Innovative Services on a Computed Tomography Scan”, In: Proceedings of MATEC Web of Conferences, 68, 18003(2016), DOI: $10.1051 /$ matecconf/20166818003.

[4] Bureau of Policy and Strategy office of the Permanent Secretary, "Progress Reports, Regional Capacity Building Framework", Available at http://bps.moph.go.th/new bps/sites/default/files/3.meeting\%20staffing\%20161159.pdf, accessed 27 July 2017.

[5] Booz AH, New products management for the 1980s, Booz, Allen \& Hamilton, Inc., New York, 1982.

[6] Rosenthal AKaSR, Integrating the Process of Product Development Initiation: Transitions to a More Manageable 'Fuzzy Front End', Sloan Management Review, winter, 1997.

[7] Koen P, M. Ajamian G, Boyce S, Clamen A, Fisher E, Fountoulakis S, et al., Fuzzy Front End: Effective Methods, Tools, and Techniques, Industrial Research, pp.5-35, 2002.

[8] G. Cooper R, "The Stage-Gate Idea-to-Launch Process-Update, What's New and NexGen Systems", Journal of Product Innovation Management, 25(3), pp.213-232, 2008.

[9] Na Ayudhya. P. I, “'Value Innovation' Strategic Product Planning”, Academic Journal of Architecture, pp.161-174, 2010.

[10] Kim JW, Lee SH, Son Y-J, Yang H-J, Chung YS, Jung H-W, "Mobile computed tomography: early experience in Korea”, Journal of Korean Neurosurgical Society, 48(1), pp.31-6, 2010. DOI: 10.3340/jkns.2010.48.1.31.

[11] Jeon JS, Lee SH, Son YJ, Yang HJ, Chung YS, Jung HW, "Mobile computed tomography : three year clinical experience in Korea", Journal of Korean Neurosurgical Society, 53(1), pp.39-42, 2013. DOI: $10.3340 / \mathrm{jkns} .2013 .53 .1 .39$.

[12] Dietrich M, Walter S, Ragoschke-Schumm A, Helwig S, Levine S, Balucani C, et al., "Is prehospital treatment of acute stroke too expensive? An economic evaluation based on the first trial", Cerebrovascular Diseases, 38(6), pp.457-463, 2014. DOI: 10.1159/000371427.

[13] Phyathai Hospital, "Mobile CT \& Stroke Treatment Unit 2017", Available at: https://www.phyathai.com/article detail/1366/th/Mobile CT \& Stroke Treatment_Unit \%E0\%B8\%AB\%E0\%B8

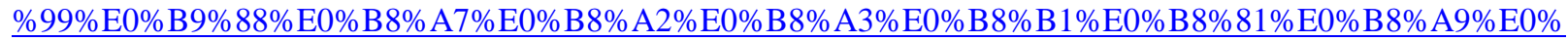
B8\%B2\%Е0\%B8\%AD\%Е0\%B8\%B1\%Е0\%B8\%A1\%Е0\%B8\%9E\%Е0\%B8\%B2\%Е0\%B8\%95\%Е0\%B9\%80\%Е 0\%B8\%89\%Е0\%B8\%B5\%E0\%B8\%A2\%Е0\%B8\%9A\%Е0\%B8\%9E\%Е0\%B8\%A5\%Е0\%B8\%B1\%Е0\%B8\%99 \% $\mathrm{E} 0 \% \mathrm{~B} 9 \% 80 \% \mathrm{E} 0 \% \mathrm{~B} 8 \% 84 \% \mathrm{E} 0 \% \mathrm{~B} 8 \% \mathrm{~A} 5 \% \mathrm{E} 0 \% \mathrm{~B} 8 \% \mathrm{~B} 7 \% \mathrm{E} 0 \% \mathrm{~B} 9 \% 88 \% \mathrm{E} 0 \% \mathrm{~B} 8 \% \mathrm{AD} \% \mathrm{E} 0 \% \mathrm{~B} 8 \% 99 \% \mathrm{E} 0 \% \mathrm{~B} 8 \%$ 97\%E0\%B8\%B5\%E0\%B9\%88, accessed 24 May 2017.

[14] Agrawal D, Saini R, Singh PK, Sinha S, Gupta DK, Satyarthee GD, et al., "Bedside computed tomography in traumatic brain injury: Experience of 10,000 consecutive cases in neurosurgery at a level 1 trauma center in India", Neurology India, 64(1), 62, 2016.

[15] John S, Stock S, Cerejo R, Uchino K, Winners S, Russman A, et al., "Brain imaging using mobile CT: current status and future prospects", Journal of Neuroimaging, 26(1), pp.5-15, 2016.

[16] Fassbender K, Grotta JC, Walter S, Grunwald IQ, Ragoschke-Schumm A, Saver JL, "Mobile stroke units for prehospital thrombolysis, triage, and beyond: benefits and challenges", The Lancet Neurology, 16(3), pp.227-37, 2017. DOI: 10.1016/s1474-4422(17)30008-x.

[17] Yamane, T, Statistics: An Introductory Analysis, Third edition, Harper and Row Publication, New York, 1973.

[18] Prasat Neurological Institute, Clinical Nursing Practice Guidelines for Stroke, Tana Press Co.,Ltd, Bangkok, pp.45, 2016.

[19] Fertikh, D., Taylor, C. R, "Head Computed Tomography Scanning: Background, Indications", Contraindications. Medscape 2013, Available at: https://emedicine.medscape.com/article/2110836-overview\#showall, accessed 21 August 2017. 